\title{
AVALIAÇÃO DO COMPORTAMENTO DA SECAGEM EM SPRAY A PARTIR DE SIMULAÇÃO DO MODELO DE LEITE INTEGRAL E DESNATADO
}

\author{
D. H. S. CHAVES ${ }^{1, a^{*}}$, P. C. A. SOUZA ${ }^{1, a}$, M. A. S. BIRCHAL ${ }^{1, b, 2}$, V. S. BIRCHAL ${ }^{1, a}$ \\ ${ }^{1}$ Universidade Federal de Minas Gerais \\ ${ }^{a}$ Departamento de Engenharia Química \\ ${ }^{\mathrm{b}}$ Departamento de Engenharia Eletrônica \\ ${ }^{2}$ Pontifícia Universidade Católica de Minas Gerais, Departamento de Engenharia de Controle e \\ Automação \\ *e-mail: diegohschaves@ outlook.com
}

\begin{abstract}
RESUMO
Este trabalho apresenta uma implementação da modelagem e um estudo por simulação do comportamento da secagem de leite integral e desnatado em regime permanente, visando-se o controle dos parâmetros operacionais para obtenção de um produto final de boa qualidade e um processo energeticamente eficiente. As equações de balanço de massa e energia foram utilizadas e avaliou-se a acoplagem de três isotermas de sorção para cada tipo de leite no modelo simulado. Dados experimentais de literatura de produção de leite em pó integral e desnatado foram usados para validar a simulação que se mostrou adequada e útil na previsão dos parâmetros operacionais do processo. Embora satisfatório, o estudo apontou modificações necessárias ao modelo preliminar que inclua equações que descrevam a cinética de secagem da partícula e uma estimativa do acúmulo de produto nas paredes de forma que este possa representar melhor o processo de secagem. O trabalho também mostra a importância de se ter um bom controle da temperatura durante o processo de secagem, variável importante para que perdas de produto durante o processo possam ser reduzidas e a eficiência energética do processo preservada. É importante ressaltar que o modelo é genérico e facilmente aplicável a qualquer outro material de propriedades conhecidas.
\end{abstract}

\section{INTRODUÇÃO}

Secagem por atomização (ou em spray, como denominada neste trabalho) é uma operação unitária bem conhecida para produção de materiais em pó a partir de soluções líquidas. Essa técnica é usada em diversos setores industriais, como alimentício, farmacêutico, cerâmico, químico, em especial os de detergentes e de pigmentos. No setor alimentício, as indústrias de laticínios são as que mais utilizam a secagem em spray na conversão do leite líquido ao leite em pó e de seus produtos derivados (MUJUMDAR, 2004).
O princípio de funcionamento da secagem em spray é a atomização da fase líquida em gotículas e sua dispersão em um fluxo de fluido quente, geralmente o ar. A atomização gera um aumento da área superficial do material a ser seco, facilitando a transferência simultânea de calor e massa, o que acarreta em um período de secagem menor com a obtenção do produto final na forma de pó (MUJUMDAR, 2014; BIRCHAL et al., 2006).

O processo de secagem em spray oferece vantagens em relação a outros processos de secagem, tais como: os produtos secos podem ser obtidos na forma de pós 
finos, grânulos ou aglomerados; estes podem ter características pré-determinadas como tamanho, densidade e teor de umidade; materiais líquidos sensíveis ao calor podem ser processados sem um dano térmico significativo para o produto, uma vez que o tempo de exposição ao ambiente quente é de curta duração; a operação é contínua e, portanto, adaptável a um controle totalmente automático, obtendo um tempo de resposta menor, além de atingir altas taxas de produção (CHEN e MUJUMDAR, 2008).

Secadores spray, em geral, necessitam de grandes quantidades de energia (JIN e CHEN, 2011). Entretanto, para se atingir boa produtividade, baixo consumo de energia e alta qualidade do produto final, é essencial o bom entendimento do processo. (MUJUMDAR, 2014; HUANG, KUMAR e MUJUMDAR, 2006). Tem-se, em literatura, um vasto estudo sobre diferentes fenômenos na secagem em spray com emprego de vários modelos matemáticos e ferramentas computacionais. Modelos teóricos consistem do estudo dos mecanismos de transferência de calor e de massa que ocorrem em regimes transiente e estacionário do secador spray (TAN, IBRAHIM e TAIP, 2010).

Embora um processo que envolva uma suspensão líquida sendo atomizada em um fluxo de ar quente possa parecer simples, projetar um secador spray industrial envolve dificuldades sérias, não apenas devido ao tamanho e escalonamento dos equipamentos, mas também pela complexidade envolvida na medição e descrição do sistema usando grande parte de fundamentos de Engenharia Química, especialmente transferência de calor e massa, assim como mecânica dos fluidos (BIRCHAL et al., 2006; LANGRISH, 2009). Sabe-se que o controle do teor de umidade é a principal variável que influencia na qualidade do produto seco (TAN, TAIP e AZIZ, 2009).

O objetivo deste trabalho é estruturar um sistema equacional que possa descrever e simular satisfatoriamente $\mathrm{o}$ processo de secagem por spray, visando analisar o desempenho geral do secador spray em função dos seus parâmetros operacionais. Particularmente, o estudo da simulação permitirá avaliar o comportamento das variáveis de saída do secador (variáveis controladas) a partir de variações nas variáveis de entrada (variáveis manipuláveis). Sabendo-se que a umidade final do pó é a variável de maior importância no processo, valores simulados dessa variável serão analisados para leite em pó desnatado e integral utilizando-se diferentes isotermas de sorção.

\section{MODELO E CONSIDERAÇÕES}

Uma abordagem da modelagem de secadores spray é descrever as equações de balanços gerais para o secador considerando seu comportamento como sendo o de um reator perfeitamente agitado (SOLTANI et al., 2014). Os balanços de massa e de energia para o secador spray permitem a estimativa da eficiência do secador e formam uma base para a concepção do mesmo, além de ajudar a minimizar as perdas térmicas e a do produto (CHEN e MUJUMDAR, 2008). O presente trabalho se baseia nas equações de balanços propostas e revisadas por Langrish (2009).

Assume-se que no interior da câmara de secagem exista uniformidade na temperatura do gás e as temperaturas de saída da fase sólida $\left(T_{s o}\right)$ e do gás $\left(T_{g o}\right)$ estão praticamente em equilíbrio, ou seja: $T_{g o}=T_{s o} \equiv T_{o}$. Entretanto, essa condição não restringe $o$ modelo, já que um offset (diferença entre os valores) entre essas duas temperaturas é facilmente inserível no procedimento de solução.

A quantidade de energia que deixa o secador spray está presente, principalmente, na fase gasosa, porém uma parte da energia é presente no produto com baixo teor de umidade. Desconsiderando-se perdas térmicas que são desprezíveis para grandes secadores 
industriais (LANGRISH, 2009), a troca de calor entre as fases pode ser assim descrita:

$$
G\left(H_{g o}-H_{g i}\right)=F\left(H_{s i}-H_{s o}\right)
$$

sendo $G$ e $F$ taxas mássicas do gas seco e do sólido seco respectivamente alimentados, $H_{g}$ e $H_{s}$ as entalpias das fases gasosa e sólida respectivamente ( $o$ e $i$ significando saída e entrada do secador). Estas entalpias podem ser calculadas pelas seguintes equações:

$$
\begin{aligned}
& H_{g}=C_{p a}\left(T_{g}-T_{r e f}\right)+Y\left[\lambda+C_{p v}\left(T_{g}-T_{r e f}\right)\right](2) \\
& H_{s}=C_{p s}\left(T_{s}-T_{r e f}\right)+X \cdot C_{p l}\left(T_{s}-T_{r e f}\right)
\end{aligned}
$$

em que a entalpia total, tanto na entrada quanto na saída, é a soma das entalpias das duas fases. Nas equações 2 e $3, C_{p}$ representa o calor especifico ( $a-$ ar, $v$ - vapor de água, $l$ - água líquida), $X$ e $Y$ umidades absolutas do sólido e do gás em base seca, $T_{\text {ref }}$ temperatura de referência $(=273,15 \mathrm{~K})$. Considerando-se que não haja perda ou acúmulo de massa no interior do secador, o balanço de massa para o secador fornece a seguinte equação:

$$
G\left(Y_{o}-Y_{i}\right)=F\left(X_{i}-X_{o}\right)
$$

Rearranjando a equação (4) para o cálculo da umidade do ar de saída, tem-se que:

$$
Y_{o}=Y_{i}+\frac{F}{G}\left(X_{i}-X_{o}\right)
$$

Empregam-se valores em base seca, que se mostram mais vantajosos uma vez que as vazões de gás e sólidos secos são as mesmas na entrada e na saída da câmara de secagem. A umidade relativa $(\psi)$ é calculada dividindo a pressão de vapor do gás $\left(P_{v}\right)$ pela pressão de vapor de saturação $\left(P_{v s a t}\right)$ à $\mathrm{T}_{\mathrm{g}}$, ou seja:

$$
\psi=\frac{p_{v}}{p_{v s a t}} \cdot 100 \%
$$

sendo que as pressões são calculadas a partir da temperatura e da umidade do gás de saída. Para calcular a pressão de vapor de saturação do ar pode-se usar a equação de Antoine expressa por:

$$
p_{v s a t}=133,3 \cdot \exp \left(18,3036-\frac{3816.44}{T_{o}\left[{ }^{\circ} C\right]+229,02}\right)
$$

sendo que $\mathrm{p}_{\text {vsat }}$ é a pressão de vapor do gás à temperatura de saída $\left(T_{o}\right)$. A umidade do gás de saída é relacionada à pressão de vapor atual pela seguinte equação:

$$
Y=0,622 \frac{p_{v}}{P_{a t m}-p_{v}}
$$

sendo necessário rearranjá-la para cálculo da pressão $p_{v}$. Logo, tem-se que:

$p_{v}=\frac{\left(Y_{o} / 0,622\right) \cdot P_{a t m}}{\left[1+\left(Y_{o} / 0,622\right)\right]}$

calculando, assim, a pressão de vapor atual do gás na saída do secador.

\section{SIMULAÇÃO DO MODELO}

As equações de 1 a 9, válidas para operação em regime permanente, são usadas para simular a secagem de leite integral e desnatado, utilizando-se um procedimento iterativo para resolução deste conjunto de equações não lineares.

$\mathrm{O}$ processo de secagem, simulado usando a plataforma virtual Matlab, permite, então, o estudo da influência das variáveis de entrada do secador nas variáveis de saída do mesmo. Figura 1 apresenta tela do simulador Matlab/Simulink. 
Figura 1 - Tela de simulação - Secagem em spray dryer

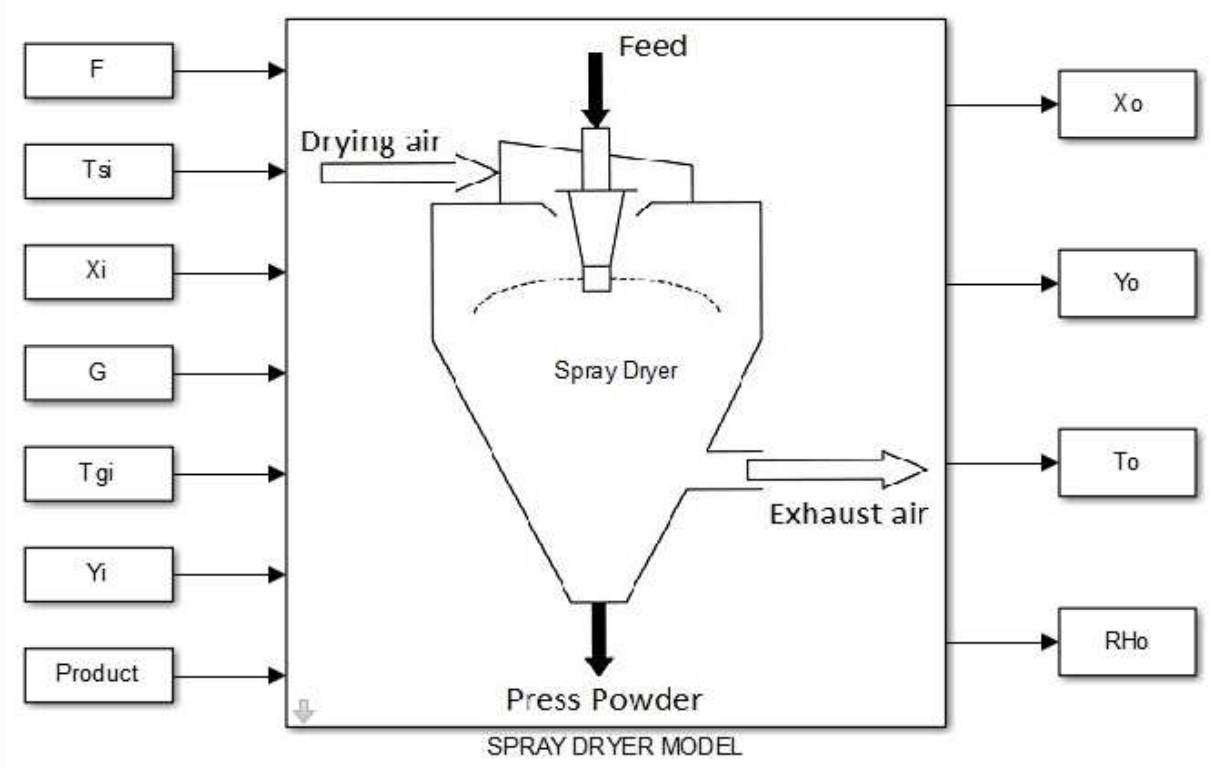

Fonte: Autor (2015).

Tabela 1 - Isotermas de sorção de leite em pó desnatado e integral.

\begin{tabular}{|c|c|c|c|}
\hline Produto & Isoterma & Equação & Referência \\
\hline \multirow{3}{*}{$\begin{array}{l}\text { Leite em } \\
\text { pó } \\
\text { desnatado }\end{array}$} & $1 \mathrm{a}$ & $X_{e q}=0,1499 \cdot \exp \left[-2,306 \times 10^{-3} \cdot T \cdot \ln \left(\frac{1}{a_{w}}\right)\right]$ & $\begin{array}{l}\text { Kockel et al. } \\
\qquad(2002)\end{array}$ \\
\hline & $1 b$ & $\begin{array}{c}X_{e q}=\frac{0,06156 \cdot C \cdot K \cdot a_{w}}{\left(1-K \cdot a_{w}\right)\left(1-K \cdot a_{w}+C \cdot K \cdot a_{w}\right)} \\
C=0,001645 \cdot \exp (2986,65 / T) \\
K=5,710 \cdot \exp (-615,59 / T)\end{array}$ & $\begin{array}{l}\text { Lin, Chen e } \\
\text { Pearse (2005) }\end{array}$ \\
\hline & $1 \mathrm{c}$ & $X_{e q}=0,0445 \frac{4,47 \cdot a_{w}}{\left(1-a_{w}\right)\left(1-a_{w}+4,47 \cdot a_{w}\right)}$ & $\begin{array}{l}\text { Murrieta-Pazos } \\
\text { et al. (2011) }\end{array}$ \\
\hline \multirow{3}{*}{$\begin{array}{l}\text { Leite em } \\
\text { pó integral }\end{array}$} & $1 d$ & $X_{e q}=0,0497 \frac{18,71 \cdot a_{w}}{\left(1-a_{w}\right)\left(1-1,13 \cdot a_{w}+17,58 \cdot a_{w}\right)}$ & $\begin{array}{l}\text { Jouppila e Roos } \\
\text { (1994) }\end{array}$ \\
\hline & 1e & $\begin{array}{c}X_{e q}=\frac{0,04277 \cdot C \cdot K \cdot a_{w}}{\left(1-K \cdot a_{w}\right)\left(1-K \cdot a_{w}+C \cdot K \cdot a_{w}\right)} \\
C=0,1925 \cdot \exp (1261,13 / T) \\
K=2,960 \cdot \exp (-386,70 / T)\end{array}$ & $\begin{array}{l}\text { Lin, Chen e } \\
\text { Pearse (2005) }\end{array}$ \\
\hline & $1 f$ & $X_{e q}=0,0244 \frac{7,34 \cdot a_{w}}{\left(1-a_{w}\right)\left(1-a_{w}+7,34 \cdot a_{w}\right)}$ & $\begin{array}{l}\text { Murrieta-Pazos } \\
\text { et al. (2011) }\end{array}$ \\
\hline
\end{tabular}


As variáveis de entrada do modelo são a vazão de sólidos secos $(F)$, a temperatura de alimentação da emulsão $\left(T_{s i}\right)$, a umidade de sólidos da alimentação $\left(X_{i}\right)$, a vazão de ar seco $(G)$, a temperatura do ar de entrada $\left(T_{g i}\right)$ e a umidade do ar de entrada $\left(Y_{i}\right)$. As variáveis de saída são a umidade de sólidos na saída $\left(X_{o}\right)$, a umidade absoluta do gás de saída $\left(Y_{o}\right)$, a temperatura de saída $\left(T_{o}\right)$ e a umidade relativa do gás de saída $\left(\psi_{o}\right)$.

$\mathrm{Na}$ simulação, pode-se avaliar o comportamento das variáveis manipuláveis para diferentes produtos e curvas de sorção, considerando-se as equações expostas na Tabela 1. Nesta Tabela 1, apresentam-se equações para a umidade de equilíbrio $\left(X_{e q}\right)$ em função da atividade de água $\left(a_{w}\right)$ e/ou da temperatura $(T)$ para o leite em pó integral e para o desnatado.

Foram utilizadas as correlações apresentadas na Tabela 2 para estimar $C_{p}$ da água, ar seco e vapor considerando que estes valores variam com a temperatura.

Tabela 2 - Calores específicos da água, ar seco e vapor em função da temperatura.

\begin{tabular}{cc}
\hline \multicolumn{2}{c}{$\mathrm{Cp}[\mathrm{kJ} / \mathrm{kgK}]$} \\
\hline Água & $4,020+5,77 \times 10^{-4} T-8,30 \times 10^{-8} T^{2}$ \\
Ar & $0,963+1,65 \times 10^{-4} T-4,59 \times 10^{2} T^{-2}$ \\
seco & (2b) \\
Vapor & $1,601+6,69 \times 10^{-4} T-5,58 \times 10^{3} T^{-2}$
\end{tabular}

Fonte: Smith, Van Ness e Abbott (2007).

Os valores de calor específico do sólido para a simulação são calculados com base na composição química dos leites em pó conforme é mostrado na Tabela 3, podendo estimar o valor com base na composição conhecida, realizando-se uma combinação linear dos seus constituintes, isto é:

$$
C_{p}=\sum x_{i} \cdot C_{p i}
$$

sabendo-se que o calor específico é uma propriedade aditiva (RAO et al., 2014).

Tabela 3 - Composição aproximada (\%) do leite integral e desnatado em pó.

\begin{tabular}{ccc}
\hline Componente & Integral & Desnatado \\
\hline Água & 2,5 & 3,2 \\
Proteína & 26,3 & 36,2 \\
Gordura & 26,7 & 0,8 \\
Carboidrato & 38,4 & 52 \\
Minerais & 6,1 & 7,8
\end{tabular}

Fonte: Fox e McSweeney (1998).

Os valores de calor específico de vários componentes de alimentos podem ser calculados por equações empíricas dispostas na Tabela 4.

Tabela 4 - Calor específico de componentes de leite e derivados.

\begin{tabular}{cc}
\hline Componente & $\mathrm{Cp}\left[\mathrm{J} / \mathrm{kg}^{\circ} \mathrm{C}\right]$ \\
\hline Proteína & $2008,2+1,2089 T-1,3129 \times 10^{-3} T^{2}$ \\
Gordura & $1984,2+1,4373 T-4,8008 \times 10^{-3} T^{2}$ \\
Carboidrato & $1548,8+1,9625 T-5,9399 \times 10^{-3} T^{2}$ \\
Minerais & $1092,6+1,8896 T-3,6817 \times 10^{-3} T^{2}$ \\
Fonte: Rao et al. $(2014)$.
\end{tabular}

\section{RESULTADOS E DISCUSSÕES}

A partir do modelo, simulações foram conduzidas empregando-se diferentes condições de entrada para determinar os efeitos nas variáveis de saída.

Observando-se o efeito da temperatura de entrada do ar na umidade final do produto, conclui-se que uma variação positiva linear na temperatura do ar de entrada gera uma variação negativa não linear na umidade do pó, conforme pode ser observado na Figura 2.

Dado que o teor de umidade do pó na saída não se comporta de maneira linear com a variação da temperatura de entrada do ar, 
não é recomendado um aumento acentuado nesta temperatura com o intuito de diminuir a umidade do produto, já que isso implicaria em um maior custo energético para obtenção de um ganho resultante não linearmente proporcional na umidade, ou seja, uma diminuição na eficiência energética do processo. Uma boa alternativa para que se atinjam os requisitos de qualidade é ajustar outras variáveis modificando, assim, as taxas de transferência de calor e de massa até que se definam os parâmetros de operação ótimos.

Figura 2 - Gráficos da temperatura do ar de entrada e umidade do pó na saída.

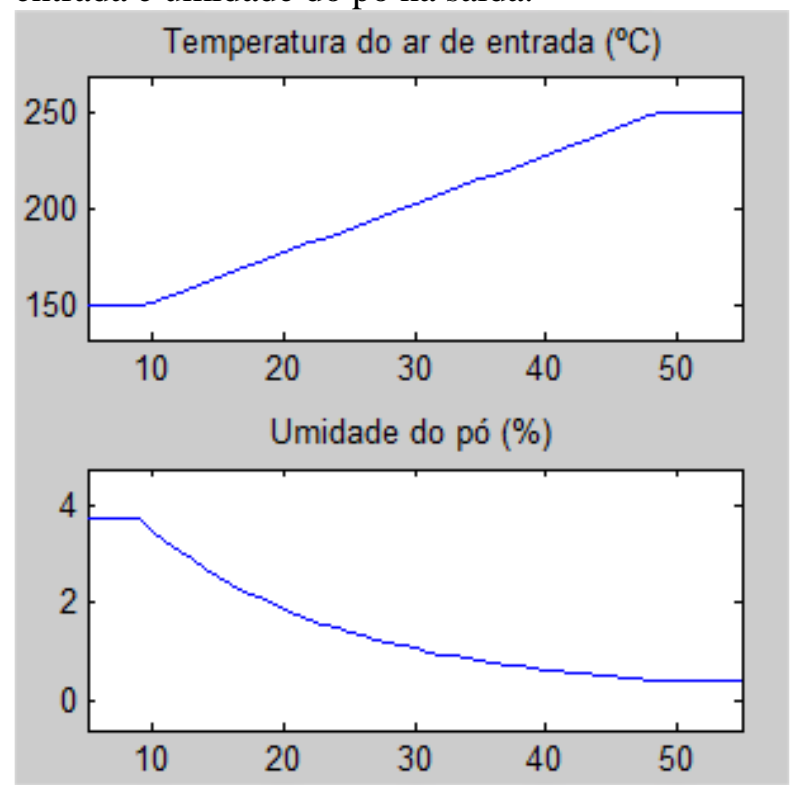

Fonte: Autor (2015).

O simulador também foi usado para analisar as saídas a partir de variações nas outras variáveis de entrada. Aplicando-se variações positivas nas vazões de ar seco e produto seco, assim como na temperatura do ar de entrada, os sinais das variações das saídas foram obtidos como mostra a Tabela 5 e encontram-se em concordância com os dados reportados na literatura (TAN, IBRAHIM e TAIP, 2010).
Tabela 5 - Variações de entrada e saída dos parâmetros do secador spray.

\begin{tabular}{cccc}
\hline & $\Delta F>0$ & $\Delta G>0$ & $\Delta T_{g i}>0$ \\
\hline$\Delta X_{o}$ & + & - & - \\
$\Delta Y_{o}$ & + & - & + \\
$\Delta T_{o}$ & - & + & + \\
$\Delta \psi_{o}$ & + & - & -
\end{tabular}

Fonte: Autor (2015).

Interpreta-se o comportamento das variáveis de saída apresentado na Tabela 5 para variações na vazão de alimentação $(\Delta F)$ como: há uma diminuição no tempo de residência no secador com o aumento da vazão, reduzindo a transferência de calor e de massa e, em consequência, $T_{o}$. A evaporação da água e a transferência do vapor para o ar é menor devido à redução do tempo de secagem, aumentando-se, então, $X_{o}, Y_{o}$ e $\psi_{\mathrm{o}}$.

O comportamento descrito na Tabela 5 para o aumento da vazão do ar de secagem está dentro do esperado. Sabe-se que um ganho nesta vazão implica em um favorecimento aos processos de transferência de massa e calor entre as fases, com diminuição em $X_{o}$ e em $Y_{o}$ em relação à vazão menor (a umidade retirada é distribuída em uma maior quantidade de massa de gás) e um aumento em $T_{o}$, também em relação a uma vazão menor, devido a um maior conteúdo energético total.

Dados experimentais obtidos na literatura foram utilizados na validação do modelo de simulação. Parâmetros de operação de secagem e valor de umidade do pó de leite desnatado foram extraídos dos trabalhos de Langrish, Marquz e Kota (2006); e os de leite integral foram retirados de Birchal et al. (2006). Os valores simulados de umidade final do pó foram, então, comparados com os experimentais reportados, conforme mostra a Tabela 6. 
Tabela 6 - Valores de umidade do pó simulados e experimentais (\% b.s.).

\begin{tabular}{cccc}
\hline Produto & Isoterma & Simulado & Experimental \\
\hline \multirow{3}{*}{ Desnatado } & $1 \mathrm{a}$ & 0,79 & \\
& $1 \mathrm{~b}$ & 0,45 & $2,62^{1}$ \\
& $1 \mathrm{c}$ & 1,96 & \\
\multirow{2}{*}{ Integral } & $1 \mathrm{~d}$ & 1,78 & \\
& $1 \mathrm{e}$ & 2,93 & $2,21^{2}$ \\
& $1 \mathrm{f}$ & 3,43 &
\end{tabular}

Fonte: Autor (2015), ${ }^{1}$ Langrish, Marquez e Kota (2006), ${ }^{2}$ Birchal et al. (2006).

Embora as isotermas do tipo BET (1c, $1 d$ e 1e) não incorporem o efeito da temperatura, sabe-se que se a umidade relativa do ar na saída estiver igual ou inferior à 0,4 , o efeito da temperatura do sólido pode ser desprezado, permitindo a extrapolação para valores de temperatura maiores que $25^{\circ} \mathrm{C}$ desse tipo de isoterma.

Percebe-se que os valores simulados da umidade do pó usando a isoterma 1c para o leite desnatado e a isoterma 1d para o leite integral foram os que mais se aproximaram dos dados experimentais. Embora estes valores simulados tenham a mesma ordem de grandeza dos experimentais, observa-se que a convergência do simulador tende para os valores de equilíbrio da umidade do pó. Tais diferenças entre os resultados experimentais e simulados ocorrem porque o modelo apresentado nesta fase preliminar ainda não contempla processos em escala laboratorial e semi-piloto, nos quais não há a ocorrência do equilíbrio térmico entre as fases na saída do secador (ou seja o tempo de residência da partícula no secador não é suficiente para garantir tal equilíbrio). Cumpre-se ressaltar que Langrish, Marquez e Kota (2006) obtiveram seus resultados experimentais para o leite desnatado em um secador em spray em escala de laboratório e Birchal et al. (2006) em um secador spray escala semi-piloto.

Para incorporar ao modelo proposto a consideração de não equilíbrio na saída do secador, faz-se necessário desacoplar, nesta saída, a temperatura do sólido da temperatura do gás. Isto acarreta mais uma variável a ser calculada, requerendo a adição de nova equação ao modelo, no caso, a descrição da cinética de secagem das gotículas de suspensão. Esta representaria as resistências à transferência de massa do sistema durante o percurso das partículas no secador.

Ressalta-se ainda que o modelo proposto neste trabalho considera que não ocorre o acúmulo de massa no interior do secador, porém, Langrish, Marquez e Kota (2006) registraram um acúmulo de aproximadamente $35 \%$ do produto nas paredes do secador, implicando, portanto, na necessidade de se introduzir um parâmetro de correção no modelo relacionado à temperatura vítrea e, consequentemente, à quantidade de produto que adere nas paredes da câmara de secagem.

O simulador é útil, então, para que se tenha um rápido estudo das especificações do produto final, podendo ser usado para estimar os parâmetros operacionais para o secador industrial que atenda às considerações preliminares deste modelo.

\section{CONCLUSÕES}

A simulação desenvolvida é capaz de descrever o comportamento, em regime permanente, de um secador spray na condição de equilíbrio térmico entre as fases em sua saída. Este modelo utiliza diferentes isotermas de sorção para descrever os processos de secagem de leite em pó integral e desnatado, juntamente com valores do calor específico de sólidos dependendo da composição do leite. Outras equações de isotermas e de calores específicos podem ser inseridas ao simulador de forma a ajustar os parâmetros operacionais para outros produtos a partir das especificações requeridas.

Identificam-se neste trabalho as modificações a serem implementadas no modelo preliminar apresentado de forma a melhor equacionar o processo de secagem 
para o leite, principalmente, no que tange à necessidade de um bom controle da temperatura do secador em spray para que as paredes da câmara de secagem não atinjam a temperatura de transição vítrea do material a ser seco. Isto gera, na prática, um depósito de material na parede do secador, ocorrendo perda de material, aumentando o consumo energético e podendo, inclusive, inviabilizar o processo.

\section{NOMENCLATURA}

$\lambda$ - Calor latente de vaporização $\left(\mathrm{kJ} \mathrm{kg}^{-1}\right)$

$\psi$ - Umidade relativa (\%)

$\psi_{o}$ - Umidade relativa do gás na saída do

secador $(\%)$

$a_{w}$ - atividade de água

$C_{p}$ - Calor específico $\left(\mathrm{kJ} \mathrm{kg}^{-1} \mathrm{~K}^{-1}\right)$

$C_{p a}$ - Calor específico do ar seco $\left(\mathrm{kJ} \mathrm{kg}^{-1} \mathrm{~K}^{-1}\right)$

$\mathrm{C}_{\mathrm{pl}}$ - Calor específico água líq. $\left(\mathrm{kJ} \mathrm{kg}^{-1} \mathrm{~K}^{-1}\right)$

$\mathrm{C}_{\mathrm{ps}}$ - Calor específico dos sólidos $\left(\mathrm{kJ} \mathrm{kg}^{-1} \mathrm{~K}^{-1}\right)$

$\mathrm{C}_{\mathrm{pv}}$ - Calor específico do vapor de água

pura $\left(\mathrm{kJ} \mathrm{kg}^{-1} \mathrm{~K}^{-1}\right)$

$F$ - Vazão de sólidos secos $\left(\mathrm{kg} \mathrm{s}^{-1}\right)$

$G$ - Vazão de ar seco $\left(\mathrm{kg} \mathrm{s}^{-1}\right)$

$H$ - Entalpia (kJ)

$H_{g}$ - Entalpia da fase gasosa $(\mathrm{kJ})$

$H_{g i}$ - Entalpia do ar na entrada do secador $(\mathrm{kJ})$

$H_{g o}$ - Entalpia do gás na saída do secador (kJ)

$H_{s}$ - Entalpia da fase sólido-úmida $(\mathrm{kJ})$

$H_{s i}$ - Entalpia dos sólidos na entrada do secador $(\mathrm{kJ})$

$H_{s o}$ - Entalpia dos sólidos na saída do secador $(\mathrm{kJ})$

$P_{\text {atm }}$ - Pressão atmosférica $(\mathrm{Pa})$

$p_{v}$ - Pressão de vapor $(\mathrm{Pa})$

$p_{v s a t}$ - Pressão de vapor de saturação $(\mathrm{Pa})$

$T$ - Temperatura $\left({ }^{\circ} \mathrm{C}\right.$ ou $\left.\mathrm{K}\right)$

$T_{g}$ - Temperatura da fase gasosa $\left({ }^{\circ} \mathrm{C}\right)$

$T_{g i}$ - Temperatura do ar na entrada do secador $\left({ }^{\circ} \mathrm{C}\right)$
$T_{g o}$ - Temperatura do gás na saída do secador $\left({ }^{\circ} \mathrm{C}\right)$

$T_{o}$ - Temperatura na saída do secador $\left({ }^{\circ} \mathrm{C}\right)$

$T_{\text {ref }}$ - Temperatura de referência $\left({ }^{\circ} \mathrm{C}\right)$

$T_{s}$ - Temperatura dos sólidos $\left({ }^{\circ} \mathrm{C}\right)$

$T_{s i}$ - Temperatura dos sólidos na entrada do secador $\left({ }^{\circ} \mathrm{C}\right)$

$T_{s o}$ - Temperatura dos sólidos na saída do secador $\left({ }^{\circ} \mathrm{C}\right)$

$x$ - fração mássica

$X$ - Umidade absoluta do produto (b.s.)

$X_{e q}$ - Umidade de equilíbrio (b.s.)

$X_{i}$ - Umidade absoluta do produto na entrada

do secador (b.s.)

$X_{o}$ - Umidade absoluta do pó na saída do

secador (b.s.)

$Y$ - Umidade absoluta do ar (b.s.)

$Y_{i}$ - Umidade absoluta do ar na entrada do secador (b.s.)

$Y_{o}$ - Umidade absoluta do gás na saída do secador (b.s.)

\section{REFERÊNCIAS}

BIRCHAL, V. S.; HUANG, L.; MUJUMDAR, A. S.; PASSOS, M. L. Spray Dryers: Modeling and Simulation. Drying Technology, v.24, p.359-371, 2006.

CHEN, X. D.; MUJUMDAR, A. S. Drying Technologies in Food Processing. Singapore: Blackwell Publishing, 2008.

FOX, P. F.; MCSWEENEY, P. L. H. Dairy Chemistry and Biochemistry. Great Britain: International Thomson Publishing, 1998.

HUANG, L. X.; KUMAR, K.; MUJUMDAR. A. S. A comparative study of a spray dryer with rotary disc atomizer and pressure nozzle using computational fluid dynamics simulations. Chemical 
Engineering and Processing, v.45, p.461470, 2006.

JIN, Y.; CHEN, X. D. Entropy production during the drying process of milk droplets in an industrial spray dryer. International Journal of Thermal Sciences, v. 50, p.615625, 2011.

JOUPPILA, K.; ROOS, Y. H. Water sorption and time-dependent phenomena of milk powders. Journal of Dairy Science, v.77, p.1798-1808, 1994.

KOCKEL, T. K.; ALLEN, S.; HENNIGS, C.; LANGRISH, T. A. G. An experimental study of the equilibrium for skim milk powder at elevated temperatures. Journal of Food Engineering, v.51, p.291-297, 2002.

LANGRISH, T. A. G. Multi-scale mathematical modeling of spray dryers. Journal of Food Engineering, v.93, p.218228, 2009.

LANGRISH, T. A. G.; MARQUEZ, N.; KOTA, K. Na investigation and quantitative assessment of particle shape in milk powders from a laboratory-scale spray dryer. Drying Technology, v.24, p.1619-1630, 2006.

LIN, S. X. Q.; CHEN, X. D.; PEARCE, D. L. Desorption isotherm of milk powders at elevated temperatures and over a wide range of relative humidity. Journal of Food Engineering, v.68, p.257-264, 2005.

MUJUMDAR, A. S. Research and development in drying: recent trends and future prospects. Drying Technology, v.22, p.1-26, 2004.

MUJUMDAR, A. S. Handbook of Industrial Drying. New York: CRC Press, 2014.
MURRIETA-PAZOS， I.; GAIANI， C.; GALET, L.; CUQ, B.; DESOBRY, S.; SCHER, J. Comparative study of particle structure evolution during water sorption: skim and whole milk powders. Colloids and Surfaces B: Biointerfaces, v.87, p.1-10, 2011.

RAO, M. A.; RIZVI, S. S. H.; DATTA, A. K.; AHMED, J. Engineering Properties of Foods. New York: CRC Press, 2014.

SMITH, J. M.; VAN NESS, H. C.; ABBOTT, M. M. Introdução à Termodinâmica da Engenharia Química. Rio de Janeiro: LTC, 2007.

SOLTANI, S.; GERDE, P.; ACEVEDO, F.; RASMUSON, A. Counter-current spray drying with stream separation: computational modeling of a novel dryer design. Chemical Engineering Research and Desing, v.93, p.163-173, 2015.

TAN, L. W.; IBRAHIM, N.; TAIP, F. S. Empirical model of spray drying of whole milk powder using nozzle atomizer spray dryer. Journal of Food, Agriculture \& Environment, v.8, p.247-252, 2010.

TAN, L. W.; TAIP, F. S.; AZIZ, N. A. Simulation and control of spray drying using nozzle atomizer spray dryer. International Journal of Engineering \& Technology, v.9, p.12-17, 2009.

\section{AGRADECIMENTOS}

Os autores agradecem à Prof. Maria Laura Passos pelas sugestões e suporte, à CAPES (Coordenação de Aperfeiçoamento de Pessoal de Nível Superior) e à FAPEMIG (Fundação de Amparo à Pesquisa do Estado de Minas Gerais) pelo suporte financeiro. 